\title{
THRESHOLD BASED FILTERING TECHNIQUE FOR EFFICIENT MOVING OBJECT DETECTION AND TRACKING IN VIDEO SURVEILLANCE
}

\author{
P.Vijayakumar ${ }^{1}$, A.V.Senthilkumar ${ }^{2}$ \\ ${ }^{1}$ Head, Department of Computer Applications, Sri Jayendra Saraswathy Maha Vidyalaya College of Arts \\ and Science, Coimbatore, Tamilnadu, India. \\ p_viji_kumar@yahoo.com \\ ${ }^{2}$ Director of MCA, Hindusthan College of Arts and Science, Coimbatore, Tamilnadu, India. \\ avsenthilkumar@yahoo.com
}

\begin{abstract}
Detection and tracking of moving objects are an important research area in a video surveillance application. Object tracking is used in several applications such as video compression, surveillance, robot technology and so on. Recently many researches has been developed for video object detection, however the object detection accuracy and background object detection in the video frames are still poses demanding issues. In this paper, a novel framework called Threshold Filtered Video Object Detection and Tracking (TFVODT) is designed for effective detection and tracking of moving objects. TFVODT framework initially takes video file as input, and then video frames are segmented using Median Filter-based Enhanced Laplacian Thresholding for improving the video quality by reducing mean square error. Next, Color Histogram-based Particle Filter is applied to the segmented objects in TFVODT framework for video object tracking. The Color Histogram-based Particle Filter measures the likelihood function, particle posterior and particle prior function based on the Bayes Sequential Estimation model for improving the object tracking accuracy. Finally, the objects detection is performed with help of Improvisation of Enhanced Laplacian Threshold (IELT) to enhance video object detection accuracy and to recognize background moving object detection. The proposed TFVODT framework using video images obtained from Internet Archive 501(c) (3) for conducting experiment and comparison is made with the existing object detection techniques. Experimental evaluation of TFVODT framework is done with the performance metrics such as object segmentation accuracy, Peak Signal to Noise Ratio, object tracking accuracy, Mean Square Error and object detection accuracy of moving video object frames. Experimental analysis shows that the TFVODT framework is able to improve the video object detection accuracy by $18 \%$ and reduces the Peak Signal to Noise Ratio by $23 \%$ when compared to the state-ofthe-art works.
\end{abstract}

Keywords: Object segmentation, Object tracking, Object Detection, Enhanced Laplacian Thresholding, Median Filter, Color Histogram-based Particle Filter

\section{INTRODUCTION}

Video surveillance systems rely on the ability to detect moving objects in the video sequence which is a relevant information mining step in a wide range of computer vision applications. Many research works has been conducted for video object detection and tracking. Self Crossing Detection for Parametric Active Contours (SCD-PAC) [1] was introduced for tracking objects in real world video sequences with the help of Sobolev active contours. However, the object detection accuracy remained unaddressed. The Automatic Estimation of Multiple Motion (AEMM) was performed in [2] using motion correspondence algorithm that automatically detects the multiple motion of object.

Visual recognition system using local invariant detectors was developed in [3] for improving the rate of features being detected with the aid of template-based descriptor. But it is not suitable for complex articulated objects. Automatic foreground object detection was presented in [4] using probabilistic consensus foreground object template for enhancing the foreground object detection results. However, foreground object cannot be perfectly detected. Multimodal analysis [5] for moving objects using Canonical Correlation Analysis (CCA) was performed to improve the accuracy of moving objects being detected. However, sounding object segmentation is not effective in some complex scenarios. An analysis of moving object detection using different image registration techniques was presented in [6].

Automatic Estimation of Multiple Motion [7] fields were performed using motion correspondence algorithm with aiming at improving the automatic identification of trajectories. A parallel histogram using particle filters [8] was designed for improving the robustness of object tracking. But, this algorithm is not robust to large variations in the appearance of the target. With the aim of removing the noise present in color video, fuzzy rules were applied [9] 
that resulted in the minimization of Mean Absolute Error (MAE) and Peak Signal-to Noise Ratio. In [10], Adaptive Appearance Model was introduced for video tracking using application dependent thresholds. However, object tracking accuracy was not at required level. The object tracking accuracy is improved in TFVODT framework by using Color Histogram-based Particle Filter.

The algorithm designed in [11] used fuzzy aggregation for object localization and robustness with intensity and texture features. Moving object segmentation method in [12] used mean shift, kalman and particle filter to improve the accuracy and robust for contour tracking. A tracking framework introduced in [13] for tracking the multiple objects based on Continuous Energy Minimization. In [14], a new approach was presented for segmenting objects in videos taken in complex scenes with multiple and different targets.

A robust segmentation method was introduced in [15] for efficient tracking of segments among various videos using least square tracking framework. Existing work on moving object representation using segmentation [16] takes into account two dominant color histograms to maintain compact and efficient object representation model. A robust algorithm was designed in [17] for automatic, noise detection and removal from moving objects in video sequences. Dynamic threshold method was introduced in [18] for moving object detection and Tracking.

Detecting and tracking robust algorithm [19] was developed for moving object of intelligent video surveillance system that optimizes the accuracy while maintaining the computational complexity low. A study of localizing target was conducted in [20] to improve detection accuracy. In [21], ELT Method was introduced for multiple moving object segmentation in video surveillance. In [22], proposed a novel Framework for Specific Object Tracking in the Multiple Moving Objects Environment. In [23], MELT method was introduced for moving object segmentation in video sequences. In [24], IELT technique initiates the process of video object segmentation, object tracking and finally object detection. Though accuracy was improved, but the mean square error incurred during object tracking remained unsolved. The mean square error rate is reduced in TFVODT framework by applying Median Filter-based Enhanced Laplacian Thresholding.

Based on the aforementioned techniques and methods presented, in this work we propose a novel framework called Threshold Filtered Video Object Detection and Tracking (TFVODT) for improving the moving object detection and tracking accuracy in video surveillance. TFVODT framework initially segment the video objects and reduce the noise frame by applying Median Filter-based Enhanced Laplacian Thresholding technique. Next, the segmented video objects are used for efficient tracking of objects using the Color Histogram-based Particle Filter. Finally, we perform an IELT for efficient Back Ground Object Detection.
The paper is organized as follows. In Section 2, we describe the design of TFVODT framework using Enhanced Laplacian Thresholding and filtering techniques. Section 3 introduces the different experimental settings studied in this work. We evaluate our algorithm for object detection and compare with the state of the work, discuss in detail in Section 4. Finally, the paper concludes in section 5.

\section{DESIGN OF THRESHOLD FILTERED VIDEO} OBJECT DETECTION AND TRACKING FRAME WORK USING ENHANCED LAPLACIAN THRES HOLDING AND FILTERING TECHNIQUES

The structure of Threshold Filtered Video Object Detection and Tracking technique is described in this section. The TFVODT framework is implemented under the Enhanced Laplacian Thresholding and filtering techniques. The TFVODT framework used two filtering techniques i.e. Median Filter-based Enhanced Laplacian Thresholding and Color Histogram-based Particle Filter for improving the video quality and reducing the noise frame. Improvisation of Enhanced Laplacian Threshold technique (IELT) is applied in TFVODT framework to perform efficient moving object detection. In TFVODT framework, the IELT technique is done with the help of Gaussian-based Neighbourhood Intensity Proportion (GNIP). The architecture diagram of Threshold Filtered Video Object Detection and Tracking technique is shown in Fig-1.

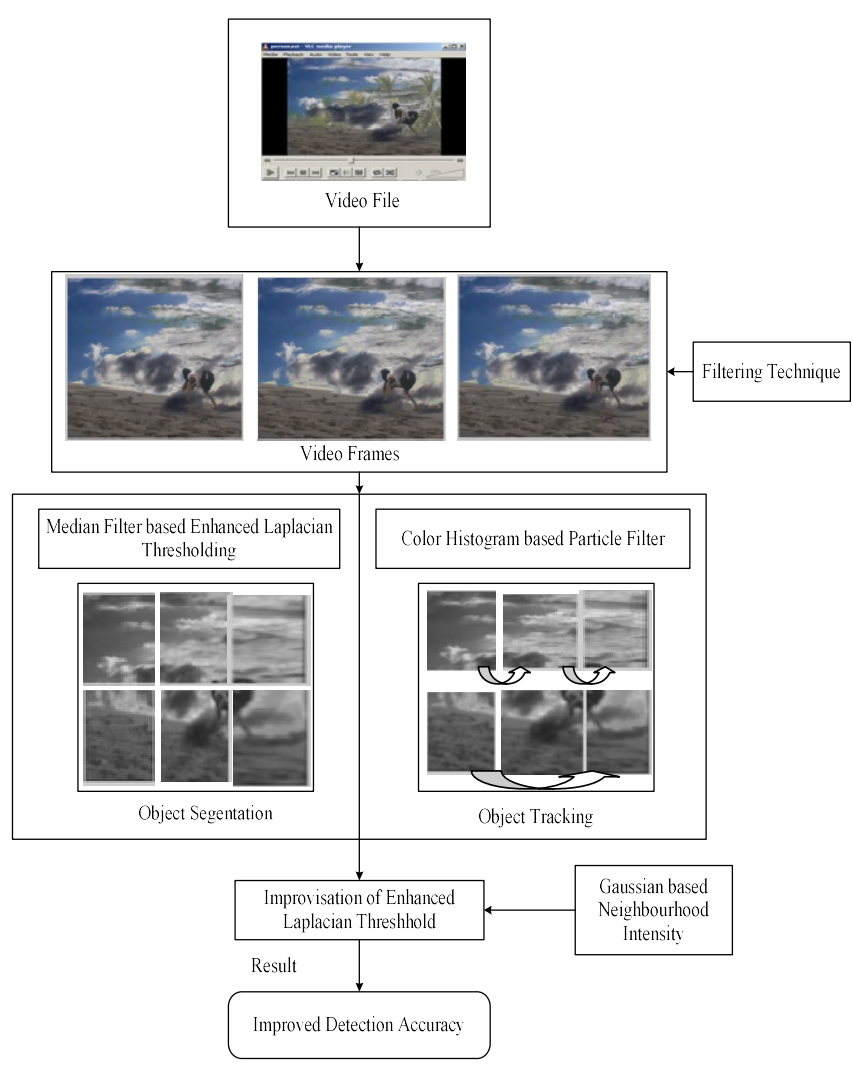

Fig-1: Architecture of Threshold Filtered Video Object Detection and Tracking technique 
As shown in Fig-1, TFVODT framework initially takes the video file as input. Next, filtering techniques are applied on the video frames for object segmentation and tracking. In TFVODT framework, two type of filtering techniques are used to remove the noise video frame and to improve the video quality. The TFVODT framework performs the object segmentation task with the help of Median Filter-based Enhanced Laplacian Thresholding technique. With the segmented object, then effective object tracking is performed based on Color Histogram-based Particle Filter that measures the likelihood function, particle posterior and particle prior function to enhance the object tracking accuracy. Finally, with the help of moving tracked objects, we perform a Gaussian-based Neighbourhood Intensity Proportion for effective moving object detection.

\subsection{Median Filter-Based Enhanced Laplacian Thres}

\section{holding for Moving Object Segmentation}

The design of TFVODT framework performs the object segmentation task with the help of Median Filter-based Enhanced Laplacian Thresholding. When an input video file is given to the TFVODT framework, video file ' $V F_{i}$ ' is partitioned into video frames ' $f$, ' All the video frames obtained do not possess characteristics such as quality, brightness or contrast. With the intention of obtaining the high quality video images without compromising the quality, brightness or contrast, preprocessing has to be performed for each input video file. Mostly several frames in video will not be of fine quality, perfect size or with good brightness and contrast.

An Un-symmetric Trimmed Median Filter is applied on the video frames with the aim of reducing the Peak Signal Noise-to Ratio and it removes the noisy frames by using a three dimension sliding window model that is mathematically formulated as,

$V F_{i}=\sum_{i=1}^{n} f_{i}(3 * 3)$

From (1), the video frame ' $V F_{i}$ ' perform denoising using ' $3 * 3$ ' sliding window. After that the median value is evaluated to replace the noisy pixels and is formulated as follows for a given ' $M_{\text {Eolwmis }} * N_{\text {rows }}$ ' of frame ' $f$, ,

$M 3 D[M * N]=$ median $\left\{\frac{M_{\text {columns }} N_{\text {rows }}}{\text { Total video frames }}\right\}$

Hierarchy-based Laplacian Thresholding is used in TFVODT framework to pre-process video frames for processing the complex video image frame by frame. The Laplacian Thresholding performs video frame fusion with the help of pattern selective model. This is because the complex video image is obtained not a pix-by-pixel, but a frame at a time. The main objective of using Laplacian Thresholding in TFVODT framework is the decomposition of thresholding on each video frames unite all these decompositions to generate a complex video image.
In TFVODT framework, The Laplacian Thresholding technique develops a thresholding for each source video frame image by applying video frame fusion for each level of thresholding with the help of frame selection decision model. The frame selection decision model selects the Most Significant Pixels (MSP) from the source video frame images, while discarding the Least Significant Pixels (LSP). The frame selection decision model is mathematically formulated as given below.

$f_{i}(M, N)=\left\{\begin{array}{l}P_{i}(M, N), \text { if }\left|\left(P_{i}(M, N)\right)\right|>\left|Q_{i}(M, N)\right| \\ Q_{i}(M, N), \text { Otherwise }\end{array}\right.$

From (3), the fused video frame image ' $f_{i}$ ' is obtained using ' $P$ ' and ' $Q$ ', the input video frames. This Frame selection decision model significantly reduces the mean square error rate in TFVODT framework. Median Filter-based Enhanced Laplacian Thresholding technique effectively segment the video objects in TFVODT framework which results in improving object segmentation accuracy.

\subsection{Color Histogram-Based Particle Filter For Moving}

\section{Object Tracking}

After segmenting the video objects, TFVODT framework using Color Histogram-based Particle Filter with aiming at improving the object tracking accuracy. The objective of using Color Histogram-based Particle Filter is to tracking of moving objects on segmented video frames. The block diagram of Color Histogram-based Particle Filter is shown in Fig-2.

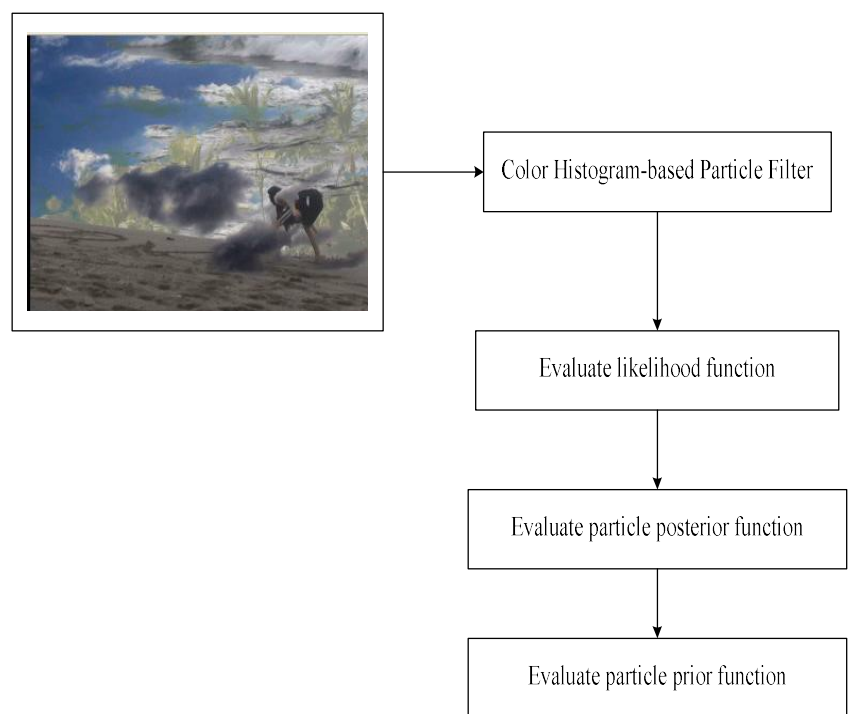

Fig-2: Block diagram of Color Histogram-based Particle Filter

As shown in figure, the Color Histogram-based Particle Filter performs three steps in TFVODT framework. The first step evaluates the likelihood function of the color histogram of the objects to be tracked. The second and third step evaluates the particles posterior and prior function with aiming at improving the object tracking accuracy. 


\subsection{Improvisation of Enhanced Laplacian Threshold}

\section{For Efficient Moving Object Detection}

Improvisation of Enhanced Laplacian Threshold techniques (IELT) is applied in our TFVODT framework with the objective of enhancing the object detection accuracy. IELT techniques preformed with the help of Gaussian-based Neighbourhood Intensity Proportion (GNIP). GNIP is used for efficient moving object detection to identify the target position in each frame by applying the Neighbourhood Intensity Proportion. The feature vectors are mined with the help of correlation in object detection phase. The TFVODT framework deals with a neighbourhood intensity proportion through incorporating the sophisticated object detection algorithm such as Gaussian Contour Foreground Video Detection. The algorithmic process of Gaussian Contour Foreground Video Detection is shown in Fig-3.

\begin{tabular}{l} 
Input: Video ' $V_{i}=V_{1}, V_{2, \ldots,} V_{n}$ ', Video Frame \\
' $V F_{i}=V F_{1}, V F_{2}, \ldots, V F_{n}$ ', weight of contour video \\
object 'Weight $t_{c}$ ', weight of foreground video object \\
'Weight', number of foreground pixels in contour \\
video object ' $N_{c}(i, j)$ ', number of contour pixels in \\
foreground video objects ' $N_{f}(i, j)$ ' \\
\hline Output: Enhanced video object detection accuracy \\
Step 1: Begin \\
Step 2: For each video frame ' $V$ ' \\
Step 3: Evaluate neighbourhood intensity proportion \\
Step 4: Measure the actual value of pixels \\
Step 5: Evaluate Neighbourhood Intensity Proportion \\
Distribution \\
Step 6: Evaluate Enhanced Moving Video Object \\
Step 7: Measure correlation coefficient \\
Step 8: End for \\
Step 9: End
\end{tabular}

Fig-3: The algorithmic of Gaussian Contour Foreground Video Detection

From the above figure, the Gaussian Contour Foreground Video Detection algorithm process is described in five steps. For each video frame, the first step evaluates the neighborhood intensity proportion to obtain the foreground video object. After that, the second step measures the actual value of pixels for removing the noise present in the video object. After removing the noise, the third step evaluates the Neighborhood Intensity Proportion Distribution to identify the foreground video object. The fourth step evaluates the Enhanced Moving Video Object Contour. Finally, the correlation coefficient is calculated with the objective of improving the moving object detection accuracy.

\section{EXPERIMENTAL SETUP}

The Threshold Filtered Video Object Detection and Tracking (TFVODT) framework conduct experimental work on MATLAB. The TFVODT framework uses the video files and their sizes are listed in Table-1 for moving object detection. The video files are obtained from Internet Archive 501(c) (3), a non-profit organization. The Internet Archive contains texts, audio, moving images, and software as well as archived web pages.

The video file information listed in table 1 contains the name of the video file, resolution of the video files and their size respectively for evaluating the TFVODT framework. The video used for moving object detection and tracking using TFVODT framework is shown below with detailed information. Experimental evaluation using TFVODT framework is conducted on various factors such as object segmentation accuracy, Peak Signal to Noise Ratio, object tracking accuracy, Mean Square Error and object detection accuracy with respect to different videos and video frames.

Table-1: Video file information

\begin{tabular}{|c|c|c|c|}
\hline \multirow[b]{2}{*}{ Name } & \multicolumn{3}{|c|}{ Video file information } \\
\hline & $\begin{array}{l}\text { Video } \\
\text { frames }\end{array}$ & Resolution & Size (KB) \\
\hline Blossom.avi & & $216 * 192$ & 349.5 \\
\hline Sample.avi & & $256 * 240$ & 113.6 \\
\hline Vehicle.avi & & $510 * 420$ & 323.7 \\
\hline Atheltic.avi & & $854 * 480$ & 905.3 \\
\hline Person.avi & & $320 * 240$ & 936.2 \\
\hline Flower.avi & & $350 * 240$ & 454.5 \\
\hline Rose.avi & & $458 * 213$ & 635.2 \\
\hline
\end{tabular}

\section{DISCUSSION}

The performance of TFVODT framework is compared with exiting two methods namely, Self Crossing Detection for Parametric Active Contours (SCD-PAC) [1], Automatic Estimation of Multiple Motion (AEMM) [2]. The performance of TFVODT framework is evaluated along with the following metrics.

\subsection{Impact of Object Segmentation Accuracy}

The object segmentation accuracy is defined as the ratio of objects being segmented to the total number of frames/second. The object segmentation accuracy is formulated as below 


$$
\mathrm{OSA}=\frac{\text { oS }}{\text { Number of frames/second }} * 100
$$

From (4), 'OSA' represents the object segmentation accuracy whereas ' $O S$ ' refers to the objects being correctly segmented. When the object segmentation accuracy is higher, the method is said to be more efficient and it is measured in terms of percentage (\%).

Table-2: Tabulation for object segmentation accuracy

\begin{tabular}{|c|c|c|c|}
\hline \multirow{2}{*}{$\begin{array}{c}\text { Number of } \\
\text { frames/second }\end{array}$} & \multicolumn{4}{|c|}{ Object segmentation accuracy (\%) } \\
\cline { 2 - 4 } & TFVODT & $\begin{array}{c}\text { SCD- } \\
\text { PAC }\end{array}$ & AEMM \\
\hline 10 & 75.25 & 66.35 & 61.54 \\
\hline 20 & 79.54 & 71.25 & 66.41 \\
\hline 30 & 84.47 & 75.68 & 70.57 \\
\hline 40 & 87.87 & 81.87 & 74.98 \\
\hline 50 & 89.17 & 84.69 & 78.94 \\
\hline 60 & 93.98 & 87.1 & 82.19 \\
\hline 70 & 95.47 & 91.81 & 86.87 \\
\hline
\end{tabular}

The object segmentation accuracy of TFVODT framework is elaborated in Table-2. We consider the framework with different number of frames/second in the range of 10 frames to 70 frames for experimental purpose using MATLAB. The performance of proposed TFVODT framework is compared with existing two methods namely, SCD-PAC [1], AEMM $[2]$.

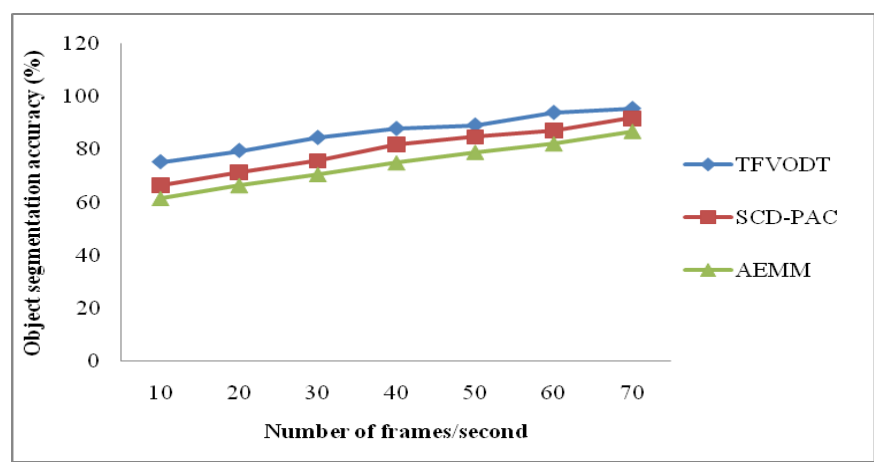

Fig-4: Measure of object segmentation accuracy

Fig-4 shows the impact of object segmentation accuracy with respect to varying frames being sent per second in the range of 10 to 70 . As illustrated in Figure, the proposed TFVODT framework performs relatively well when compared to two other methods SCD-PAC, AEMM. This is because of the application of Median Filter-based Enhanced Laplacian Thresholding that efficiently removes the noise frame for improving the video quality. Therefore, the object segmentation accuracy using TFVODT framework is improved by $7.95 \%$ as compared to SCD-PAC [1] and $14.12 \%$ as compared to AEMM [2] respectively.

\subsection{Impact of Peak Signal To Noise Ratio}

Peak Signal-to-Noise Ratio calculates the ratio between the reference video frame and the distorted video frame being detected in a video file. The higher the PSNR, the closer the distorted video frame is to the original. Therefore, higher
PSNR value associate with higher quality image (i.e. detected image) and is mathematically formulated as given below.

$$
\begin{aligned}
& M S E=\sum_{i=1}^{n}\left(V_{i}-V_{i}^{v}\right) \\
& P S N R=10 \log 10 \frac{R^{2}}{M S E}
\end{aligned}
$$

From (5), the mean square error ' $M S E$ ' is the difference between the actual frame size ' $\boldsymbol{V}_{i}$ ' and the estimated frame size ' $V_{i}^{j}$ ' being detected. From (6), the peak signal-to-noise ratio ' $P S N R$ ' is evaluated using the unsigned integer data type (with size 255) with respect to mean square error rate 'MSE' respectively.

Table-3: Tabulation for PSNR

\begin{tabular}{|c|c|c|c|}
\hline \multirow{2}{*}{$\begin{array}{c}\text { Size of } \\
\text { video file } \\
\text { (KB) }\end{array}$} & \multicolumn{3}{|c|}{ Peak Signal to Noise Ratio (\%) } \\
\cline { 2 - 4 } & TFVODT & SCD-PAC & AEMM \\
\hline 113.6 & 26.65 & 20.35 & 16.32 \\
\hline 323.7 & 31.54 & 25.13 & 20.54 \\
\hline 349.5 & 37.54 & 30.03 & 24.65 \\
\hline 454.5 & 39.87 & 32.19 & 28.98 \\
\hline 635.2 & 42.51 & 35.43 & 32.14 \\
\hline 905.3 & 44.65 & 38.15 & 36.11 \\
\hline 936.2 & 47.87 & 41.82 & 40.21 \\
\hline
\end{tabular}

To determine the performance of the PSNR for moving object detection, comparison is made with two other existing methods SCD-PAC [1], AEMM [2]. In Fig-5, the size of video frames is varied between $100 \mathrm{~KB}$ and $1000 \mathrm{~KB}$. From the figure it is illustrative that the PSNR using the proposed TFVODT framework is reduced when compared to existing methods.

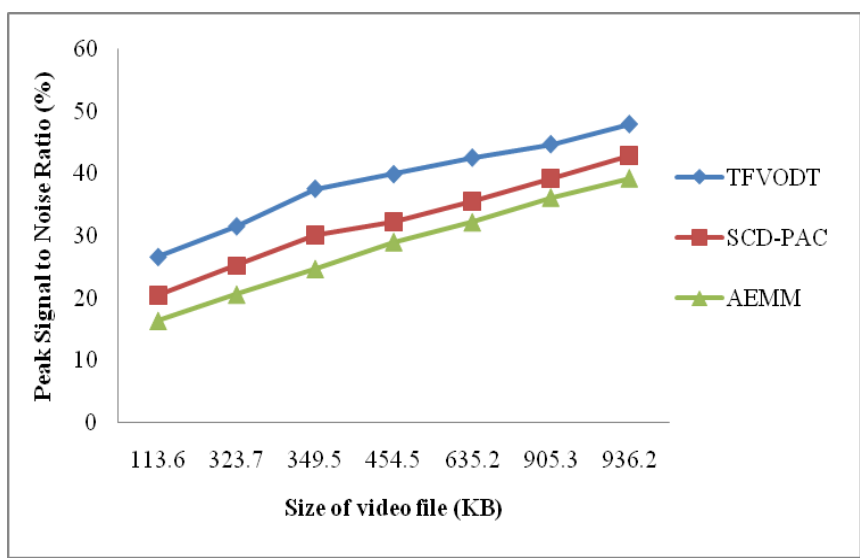

Fig-5: Measure of Peak Signal to Noise Ratio

The PSNR rate for moving object detection is reduced by applying Color Histogram-based Particle Filter in TFVODT framework. With the application of Color Histogram-based Particle Filter, posterior function and prior function is evaluated for each video frames in an iterative manner that provides the results with respect to varying frame size which in turn reducing the PSNR by $18.15 \%$ as compared to SCDPAC [1]. In addition, by applying the state space 
representation model between the state space and current measurement, by formulating an initial posterior function minimizes the PSNR for moving object detection by $27.83 \%$ as compared to AEMM [2].

\subsection{Impact of Object Tracking Accuracy}

The object tracking accuracy is defined as the ratio of objects being tracked to the total number of frame / second. The object tracking accuracy is formulated as below

$$
\text { OT } A=\frac{\text { oT }}{\text { Number of frames/sacond }}=100
$$

From (7), 'OTA' signifies the object tracking accuracy whereas ' $O T$ ' refers to the objects being correctly tracked. When the object tracking accuracy is higher, the method is said to be more efficient and it is measured in terms of percentage $(\%)$.

Table-4: Tabulation for object tracking accuracy

\begin{tabular}{|c|c|c|c|}
\hline \multirow{2}{*}{$\begin{array}{c}\text { Number of } \\
\text { frames/second }\end{array}$} & \multicolumn{3}{|c|}{ Object tracking accuracy (\%) } \\
\cline { 2 - 4 } & TFVODT & $\begin{array}{c}\text { SCD- } \\
\text { PAC }\end{array}$ & AEMM \\
\hline 10 & 77.22 & 74.83 & 71.24 \\
\hline 20 & 81.65 & 78.21 & 75.64 \\
\hline 30 & 86.25 & 83.31 & 80.87 \\
\hline 40 & 79.73 & 76.29 & 73.69 \\
\hline 50 & 84.89 & 81.16 & 79.34 \\
\hline 60 & 87.32 & 84.89 & 81.27 \\
\hline 70 & 90.78 & 87.32 & 85.11 \\
\hline
\end{tabular}

The object tracking accuracy for multiple moving object tracking using TFVODT, SCD-PAC, and AEMM is elaborated in Table-4. We consider the framework with different number of frames/second in the range of 10 frames to 70 frames for experimental purpose using MATLAB.

In Fig-6, the object tracking accuracy using the proposed TFVODT framework is higher when compared to two other existing methods SCD-PAC [1], AEMM [2]. With respect to the increasing number of videos and frames/second, the object tracking accuracy is increased though not observed to be linear (i.e. due to the presence of noise), but shows gradual improvement by using the Color Histogram-based Particle Filter when compared to SCD-PAC, AEMM.

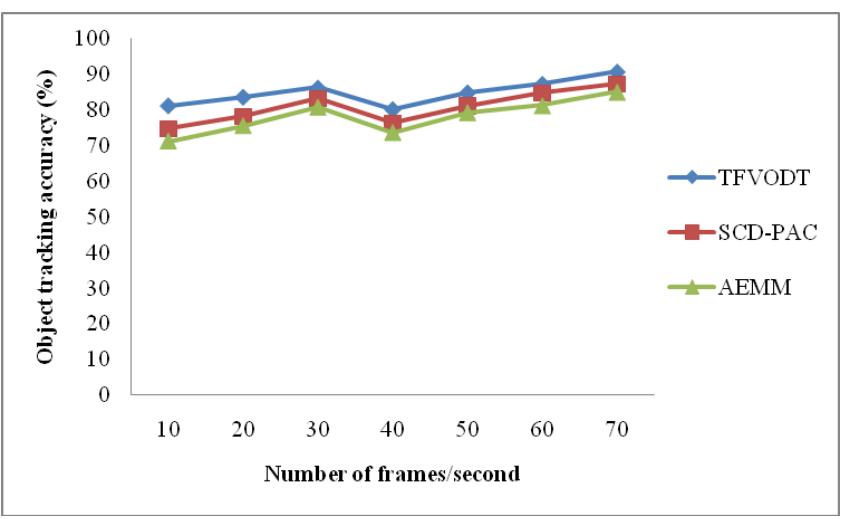

Fig-6: Measure of Object tracking accuracy
Fig-6 illustrates the rate of object tracking accuracy and is observed to be higher using TFVODT framework. The rate of object tracking accuracy is verified for different number of frames. By applying Color Histogram-based Particle Filter, the object tracking accuracy is improved using TFVODT framework by $4.80 \%$ as compared to SCD-PAC [1]. In addition, by evaluating the likelihood function of the color histogram in TFVODT framework, the object tracking accuracy is improved by $7.98 \%$ as compared to AEMM [2].

\subsection{Impact of Mean Square Error}

The Mean Square Error (MSE) in TFVODT framework is used to measure the video quality being obtained during multiple object tracking. The MSE represents the error between the tracked frames and the original video frame. When lower the value of MSE, the method is said to be more efficient and it is measured in terms of $\mathrm{db}$.

$$
M S E=\sum_{i=1}^{n}\left(V_{i}-V_{i}\right)
$$

From (8), the mean square error ' $M S E$ ' is the difference between the actual frame size ' $\boldsymbol{V}_{i}$ ' and the estimated frame size ' $V_{i}^{\prime}$ ' being tracked.

Table-5: Tabulation for mean square error

\begin{tabular}{|c|c|c|c|}
\hline \multirow{2}{*}{$\begin{array}{c}\text { Size of video } \\
\text { frame (KB) }\end{array}$} & \multicolumn{3}{|c|}{ Mean Square Error (db) } \\
\cline { 2 - 4 } & TFVODT & $\begin{array}{c}\text { SCD- } \\
\text { PAC }\end{array}$ & AEMM \\
\hline 113.6 & 21.63 & 25.34 & 29.24 \\
\hline 323.7 & 23.87 & 26.44 & 30.87 \\
\hline 349.5 & 25.98 & 30.22 & 35.47 \\
\hline 454.5 & 30.21 & 32.77 & 37.59 \\
\hline 635.2 & 37.34 & 39.35 & 44.88 \\
\hline 905.3 & 43.87 & 46.25 & 49.14 \\
\hline 936.2 & 51.57 & 55.35 & 57.68 \\
\hline
\end{tabular}

Table- 5 represents the mean square error rate with respect to different size of video frame using MATLAB and comparison is made with two other methods, namely SCDPAC [1], AEMM [2].

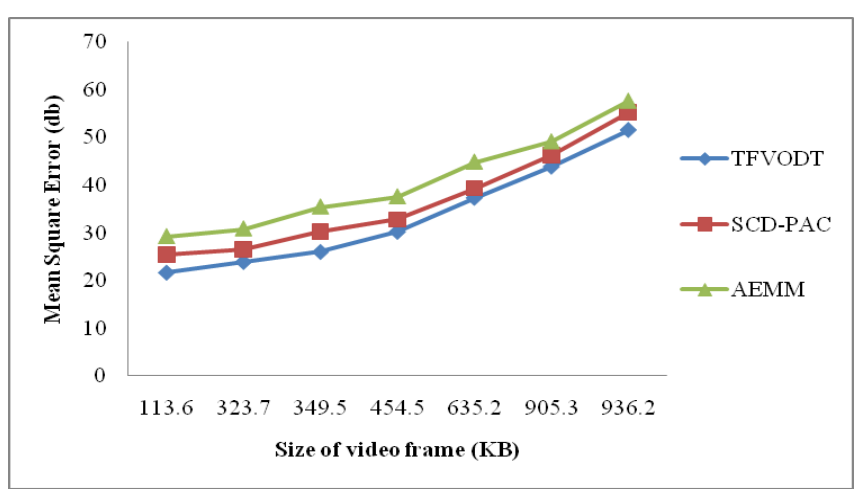

Fig-7: Measure of Mean Square Error

Fig-7 shows the mean square error rate with size of the video frame taken as the input for moving object detection. As illustrated in Figure, the proposed TFVODT framework provides minimum mean square error rate when compared 
to two other methods SCD-PAC, AEMM. This is because of the application of Median Filter-based Enhanced Laplacian Thresholding technique in TFVODT framework. With the application of Median Filter-based Enhanced Laplacian Thresholding, Bayes estimation of posterior and prior function is evaluated in an efficient manner. This in turn helps in reducing the mean square error for moving object detection using TFVODT framework by $10.12 \%$ as compared to SCD-PAC [1]. In addition, the TFVODT framework using Bayes Sequential Estimation that takes state space as input helps in decreasing the mean square error by $24.22 \%$ as compared to AEMM [2].

\subsection{Impact of Object Detection Accuracy}

The object detection accuracy is defined as the ratio of objects being detected to the total number of frames/second. The object detection accuracy is mathematically formulated as below

$$
O D A=\frac{O D}{\text { Number of frames/secord }} 100
$$

From (9), ' $O D A$ ' signifies the object detection accuracy whereas ' $O D$ ' refers to the objects being correctly detected. When the object detection accuracy is higher, the method is said to be more efficient and it is measured in terms of percentage $(\%)$.

Table-6: Tabulation for object detection accuracy

\begin{tabular}{|l|l|}
\hline Methods & $\begin{array}{l}\text { Object detection accuracy } \\
\text { (\%) }\end{array}$ \\
\hline TFVODT & 88.35 \\
\hline SCD-PAC & 75.12 \\
\hline AEMM & 69.31 \\
\hline
\end{tabular}

The object detection accuracy for TFVODT framework is elaborated in Table- 6 and comparison made with two other methods SCD-PAC, AEMM respectively. We consider the method with seven video objects and different number of frames/second in the range of 10 frames to 70 frames for experimental purpose using MATLAB.

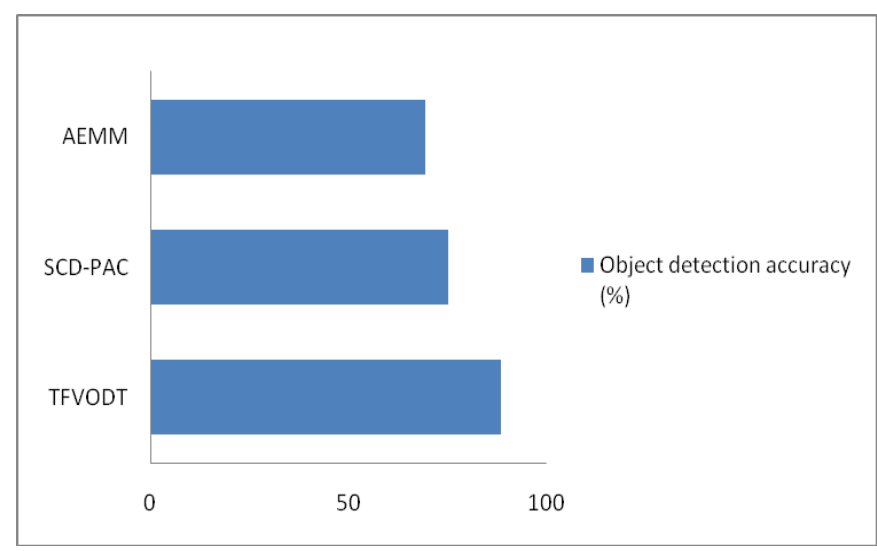

Fig-8: Measure of object detection accuracy

From the Fig-8, it is illustrative that the object detection accuracy using the Improvisation of Enhanced Laplacian
Threshold techniques in TFVODT framework is higher than when compared to the existing methods SCD-PAC, AEMM method respectively. This is because of the application of Gaussian mixture model with neighbourhood intensity proportion in TFVODT framework that measures the correlation coefficient of the moving video object in an efficient manner using Gaussian Contour Foreground Video Detection algorithm with Neighbourhood Intensity Proportion Distribution. Therefore, the object detection accuracy is improved by $14.97 \%$ when compared to SCDPAC [1] method and $21.55 \%$ improvement when compared to AEMM [2] respectively.

\section{CONCLUSION}

In this work, an effective novel framework is designed called as Threshold Filtered Video Object Detection and Tracking (TFVODT) for effective detection of moving objects in video frames. The TFVODT technique improves the object segmentation accuracy with reduced PSNR by applying Median Filter-based Enhanced Laplacian Thresholding technique. The goal of TFVODT technique for video surveillance is to improve the object segmentation accuracy, object tracking accuracy and object detection accuracy by demonstrating with training and test video objects obtained from Internet Archive 501(c) (3), a nonprofit organization which significantly contributes to the relevance. With the experiments conducted for TFVODT technique, it is observed that of video object detection for different video samples provided more accurate results as compared to existing detection methods. The results show that TFVODT technique provides better performance with an improvement of object detection accuracy by $18 \%$ and reduces the PSNR rate by $23 \%$ when compared to state of the art works.

\section{REFERENCES}

[1]. Arie Nakhmani, Allen Tannenbaum, "Self-Crossing Detection and Location for Parametric Active Contours", IEEE Transactions on Image Processing, Volume 21, Issue 7, July 2012, Pages 3150 - 3156.

[2]. Manya V. Afonso, Jacinto C. Nascimento and Jorge S. Marques, "Automatic Estimation of Multiple Motion Fields From Video Sequences Using a Region Matching Based Approach", IEEE Transactions on Multimedia, Volume 16, Issue: 1, January 2014, Pages $1-14$.

[3]. Taehee Lee, Stefano Soatto, "Video-based descriptors for object recognition", Elsevier, Image and Vision Computing, Volume 29, Issue 10, September 2011, Pages 639-652.

[4]. Shih-Wei Sun, Yu-Chiang Frank Wang, Fay Huang, Hong-Yuan Mark Liao, "Moving foreground object detection via robust SIFT trajectories", Journal of Visual Communication and Image Representation, Volume 24, Issue 3, April 2013, Pages 232-243.

[5]. Hamid Izadinia, Imran Saleemi, and Mubarak Shah, "Multimodal Analysis for Identification and Segmentation of Moving-Sounding Objects", IEEE Transactions on Multimedia, Volume 15, Issue 2, 
February 2013, Pages 378-390.

[6]. Subhabrata Bhattacharya, Haroon Idrees, Imran Saleemi, Saad Ali and Mubarak Shah, "Moving Object Detection and Tracking in Forward Looking Infra-Red Aerial Imagery", Springer, Machine Vision Beyond Visible Spectrum, May 2011, Pages 1-32.

[7]. Manya V. Afonso, Jacinto C. Nascimento and Jorge S. Marques, "Automatic Estimation of Multiple Motion Fields From Video Sequences Using a Region Matching Based Approach", IEEE Transactions on Multimedia, Volume 16, Issue: 1, January 2014, Pages $1-14$.

[8]. Henry Medeiros, Germán Holguín, Paul J. Shin, Johnny Park, "A parallel histogram-based particle filter for object tracking on SIMD-based smart cameras", Elsevier, Volume 114, Issue 11, November 2010, Pages 1264-1272.

[9]. Tom Mélange, Mike Nachtegael, and Etienne E. Kerre, "Fuzzy Random Impulse Noise Removal From Color Image Sequences", IEEE Transactions on Image Processing, Volume 20, Issue 4, April 2011, Pages 959-970.

[10]. Samuele Salti, Andrea Cavallaro, Luigi Di Stefano, "Adaptive Appearance Modeling for Video Tracking: Survey and Evaluation", IEEE Transactions on Image Processing, Volume 21, Issue 10, October 2012, Pages 4334-4348.

[11].Pojala Chiranjeevi and Somnath Sengupta, "Neighborhood Supported Model Level Fuzzy Aggregation for Moving Object Segmentation", IEEE Transactions on Image Processing, Volume 23, Issue 2, February 2014, Pages 645 - 657.

[12].Barga Deori and Dalton Meitei Thounaojam, "A Survey on Moving Object Tracking in Video", International Journal on Information Theory (IJIT), Vol.3, No.3, July 2014, Pages $31-46$.

[13]. Sukanyathara J and Alphonsa Kuriakose, "An Optimized Framework for Detection and Tracking of Video Objects in Challenging Backgrounds", The International Journal of Multimedia \& Its Applications (IJMA), Vol.6, No.4, August 2014, Pages 27 - 38.

[14].Daniela Giordano, Francesca Murabito, Simone Palazzo, Concetto Spampinato and University of Catania, "Superpixel-based Video Object Segmentation using Perceptual Organization and Location Prior", The IEEE Conference on Computer Vision and Pattern Recognition (CVPR), 2015, Pages 4814-4822.

[15].Zhengyang Wu, Fuxin Li, Rahul Sukthankar and James M. Rehg, "Robust Video Segment Proposals with Painless Occlusion Handling", The IEEE Conference on Computer Vision and Pattern Recognition (CVPR), Computer Vision Foundation, 2015, Pages 4194 4203.

[16].Jianping Han, Tian Tan, Longfei Chen and Daxing Zhang, "Moving Objects Representation for Object Based Surveillance Video Retrieval System", International Journal of Security and Its Applications, Vol.8, No.2, March 2014, Pages 315 - 322.

[17]. Safvan Vahora, Narendra Chauhan, Nilesh Prajapati, "A Robust Method for Moving Object Detection Using
Modified Statistical Mean Method", International Journal of Advanced Information Technology (IJAIT), Vol. 2, No.1, February 2012, Pages 65-73.

[18].Ms Jyoti J. Jadhav, "Moving Object Detection and Tracking for Video Survelliance", International Journal of Engineering Research and General Science Vol. 2, No. 4, July 2014, Pages 372-378.

[19].Mrinali M. Bhajibhakare, Pradeep K. Deshmukh, “To Detect and Track Moving Object for Surveillance System", International Journal of Innovative Research in Computer and Communication Engineering, Vol. 1, No. 4, June 2013, Pages 945-949.

[20].R. Kwitt1, N. Vasconcelosb, S. Razzaquec, S. Aylward, "Localizing Target Structures in Ultrasound Video -A Phantom Study", Elsevier, Volume 17, Issue 7, October 2013, Pages 712-722.

[21].P.Vijayakumar and A.V.Senthil Kumar "Moving Object Segmentation using Enhanced Laplacian Thresholding Method for Video Surveillance",IJCA,Volume 100,Issue 4,August 2014,Pages $13-17.1$

[22].P.Vijayakumar and A.V.Senthil Kumar "A Novel Framework for Specific Object Tracking in the Multiple Moving Objects Environment",IJAER,Volume 10, Number 16 (2015) Pages 36540 - 36545.

[23].P.Vijayakumar and A.V.Senthil Kumar "Moving Object Segmentation Using Median Filter

[24].-Based Enhanced Laplacian Thresholding "EJSR, Volume 133,Issue Pages 415- 427.

[25].P.Vijayakumar and A.V.Senthil Kumar" Improvising Enhanced Laplacian Thresholding Technique for Efficient Moving Object Detection in Video Surveillance" IOSRJEN, Vol. 06, Issue 01 (January. 2016), ||V1\| PP 42-52. 